ARTICLE

Received 21 Apr 2016 | Accepted 27 Jun 2016 | Published 10 Aug $2016 \quad$ DOl: 10.1038/ncomms12374

\title{
Ultrafast formation of air-processable and high-quality polymer films on an aqueous substrate
}

Jonghyeon Noh ${ }^{1, \star}$, Seonju Jeong ${ }^{1, \star} \&$ Jung-Yong Lee ${ }^{1}$

Polymer solar cells are attracting attention as next-generation energy sources. Scalable deposition techniques of high-quality organic films should be guaranteed to realize highly efficient polymer solar cells in large areas for commercial viability. Herein, we introduce an ultrafast, scalable, and versatile process for forming high-quality organic films on an aqueous substrate by utilizing the spontaneous spreading phenomenon. This approach provides easy control over the thickness of the films by tuning the spreading conditions, and the films can be transferred to a variety of secondary substrates. Moreover, the controlled Marangoni flow and ultrafast removal of solvent during the process cause the films to have a uniform, high-quality nanomorphology with finely separated phase domains. Polymer solar cells were fabricated from a mixture of polymer and fullerene derivatives on an aqueous substrate by using the proposed technique, and the device exhibited an excellent power conversion efficiency of $8.44 \%$. Furthermore, a roll-to-roll production system was proposed as an air-processable and scalable commercial process for fabricating organic devices.

\footnotetext{
${ }^{1}$ Graduate School of Energy, Environment, Water, and Sustainability (EEWS), Graphene Research Center, Korea Advanced Institute of Science and Technology (KAIST), Daejeon 305-701, Republic of Korea. * These authors contributed equally to this work. Correspondence and requests for materials should be addressed to J-Y.L. (email: jungyong.lee@kaist.ac.kr).
} 
C ontrol of the nanomorphology in polymer solar cells (PSCs) is a key factor for maximizing the module power conversion efficiency (PCE) and enhancing the electrical properties related to exciton dissociation at the interfaces between donors and acceptors ${ }^{1-3}$, diffusion of charge carriers ${ }^{4,5}$, and charge collection at each electrode $e^{6-8}$. The nanomorphology of the blended film can be engineered via thermal and solvent annealing ${ }^{9,10}$ or the use of processing additives and so on, to achieve selective solubility ${ }^{11}$. Effective manipulations may favorably influence the crystallization and orientation of the polymers $^{12}$.

Although spin-coating is the most appropriate laboratory-scale film formation process, scale-up of this process does not ensure uniformity, even within a device. This makes the spin-coating process incompatible with roll-to-roll (R2R) production under ambient conditions, where the latter is desirable for successful commercialization.

Furthermore, air processability of PSCs is a critical issue given that an inert environment is currently required for the entire process, unless the devices are stable under ambient conditions. Although poly(3-hexylthiophene) (P3HT)-based devices exhibit good air processability due to reversible degradation by oxygen, highly efficient polymers containing the benzodithiophene (BDT) group, such as poly[[4,8-bis[(2-ethylhexyl)oxy]benzo[1,2-b:4,5-b'] dithiophene-2,6-diyl][3-fluoro-2-[(2-ethylhexyl) carbonyl]thieno [3,4-b]thiophenediyl]] (PTB7) and poly[4,8-bis(5-(2-ethylhexyl) thiophen-2-yl)benzo[1,2-b;4,5-b']dithiophene-2,6-diyl-alt-(4-(2ethylhexyl)-3-fluorothieno[3,4-b]thiophene-)-2-carboxylate-2-6diyl)] (PTB7-Th), tend to undergo significant degradation on exposure to oxygen and light ${ }^{13-16}$.

Here we propose a new approach to form high-quality organic films on an aqueous (water based) substrate under ambient conditions. A drop of organic material blended into a solvent, when dropped onto an aqueous surface, spreads spontaneously and rapidly by the Marangoni effect ${ }^{17}$, leading to the rapid and uniform formation of an organic layer that can be functionalized on various substrates once transferred. Interestingly, the nanomorphology of blended films can be efficiently managed in seconds during the process of spreading on the aqueous substrate. We verify that the ultrafast solvent removal by the spontaneous spreading process can impede the infiltration of oxygen into the film via the remaining solvent effectively.

The utility of the films in functional devices is demonstrated by applying the new film formation technique to the fabrication of highly efficient PSCs. PTB7-based PSCs are successfully fabricated by exploiting the high-quality nanomorphology within the bulk heterojunction (BHJ) films. Last, we demonstrate the potential of our method as a scalable process by transferring the spontaneously spreading (SS) film from water surface to a large plastic substrate using a custom-made R2R process.

\section{Results}

Spontaneously spreading process of polymer solutions. When chemical agents with low surface energy (for example, hydrocarbon solvents or detergents) are dropped into high-surfaceenergy media (for example, water), local surface tension gradients occur on the boundary between the surrounding materials, leading to surficial flow toward regions of higher surface tension. This spontaneous spreading is called Marangoni flow ${ }^{17,18}$. The spreading coefficient $(S)$ dictates the speed of the spreading flow and is defined by the surface tensions at the three-phase contact line between a liquid droplet and liquid substrates ${ }^{19}$, where $S=\gamma_{1}-\gamma_{2}-\gamma_{12}\left(\gamma_{1}\right.$ and $\gamma_{2}$ are the surface tensions of the base and polymer solutions, respectively, and $\gamma_{12}$ is the interfacial surface tension of the two solutions). Figure 1a illustrates the different behaviours of the polymer solution depending on $S$ when a drop of polymer solution is dropped on the base solution. The flow of the solution is determined by the net force direction of the surface tensions. If $S$ is positive, that is, the surface tension of the base solution, $\gamma_{1}$ (red arrow), is larger than the sum of $\gamma_{2}$ (green arrow) and $\gamma_{12}$ (blue arrow), the polymer solution will spread over the aqueous substrate surface and form a uniform polymer film. Otherwise, the polymer solution will ball on the aqueous substrate without dispersion.

The proportion $S^{1 / 2}$ describes the spreading speed of the solution ${ }^{20,21}$, and the solution is unlikely to spread if $S$ is negative. The spreading coefficients of 1,2-dichlorobenzene $(o-\mathrm{DCB})$ and chlorobenzene (CB) are -4.25 and 2.35 dyne $^{-1}$, respectively ${ }^{21,22}$; thus only $\mathrm{CB}$ would exhibit spontaneous spreading flow on water. Dissolution of the solvent in water further accelerates drying and formation of the polymer films on aqueous substrates. Figure $1 \mathrm{~b}$ illustrates the formation of a SS film on an aqueous substrate (here water is used as the aqueous substrate). (I) The polymer solution is dropped onto the aqueous substrate; (II) the solution quickly spreads (if $S>0$ ) over the aqueous surface within a few seconds; (III) because the solvents evaporate and dissolve into the liquid simultaneously, the drying process occurs instantaneously to form a thin solid polymer layer (the SS film) on the liquid; (IV) this thin film can readily be transferred to a variety of target substrates, for example, by stamping the substrates on the SS film gently (see Supplementary Movie 1, Supplementary Note 1 and Supplementary Fig. 1).

Because the photoactive films of polymer solar cells have dimensions below the wavelengths of visible light $(<\sim 200 \mathrm{~nm})$, control of the thickness within tens of nanometers is very important for achieving optimized performance ${ }^{23}$. Herein, the SS film formation method is shown to be extremely reliable for controlling the thickness of polymer films over a wide range (tens to hundreds of nanometers). The ultimate thickness of organic films forming on water depends on the volume and concentration of the polymer solution, the size of the water bath, and the number of layers transferred (see Supplementary Note 2 and Supplementary Fig. 2a-d). PTB7:PC ${ }_{71} \mathrm{BM}$ solution was used for the thickness experiments.

Uniform and conformal transfer on various substrates. The SS films formed in the water bath exhibited controllable thickness and high uniformity over large areas. The SS-PTB7:PC ${ }_{71} \mathrm{BM}$ film was deposited in a square PET Petri dish $\left(12 \times 12 \mathrm{~cm}^{2}\right)$, leading to a thickness of $43.9 \pm 4.1 \mathrm{~nm}$ (see Supplementary Note 3 with Supplementary Figs 3 and 4) as determined from 16 positions on the substrate.

The SS films were also successfully transferred to various secondary substrates including polydimethylsiloxane (PDMS), polyethylene terephthalate (PET), paper, and the curved surface of a vial by the stamping method (Fig. 1c-f). An interference pattern was still apparent on the polyurethane (PU) substrate with sinusoidal gratings (period: $556 \mathrm{~nm}$ ) after transfer of the SS films to the substrate (Supplementary Note 4; Supplementary Fig. 5a,b). Scanning electron microscope (SEM) images also confirmed successful formation of the SS films.

Nanomorphology within BHJ films. The universality of SS film formation was demonstrated by generating the SS films using several types of common polymers. AFM images (Supplementary Note 5 and Supplementary Fig. 6) show that poly(3-hexylthiophene-2,5-diyl) (P3HT), poly[2-methoxy-5-(2-ethylhexyloxy)-1,4-phenylenevinylene] (MEH-PPV), PTB7, and PTB7-Th formed uniform and smooth SS films. The SS phenomenon 
a
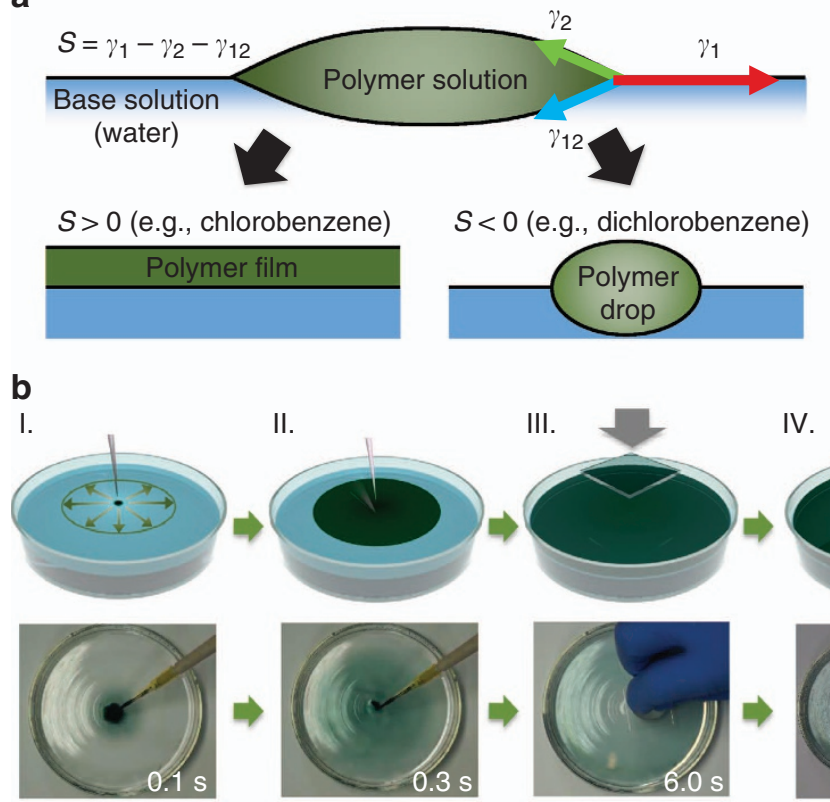

II.
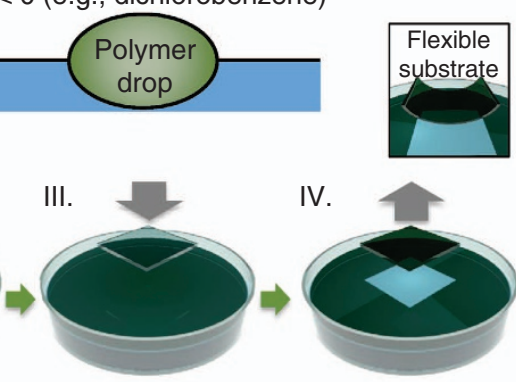

IV.
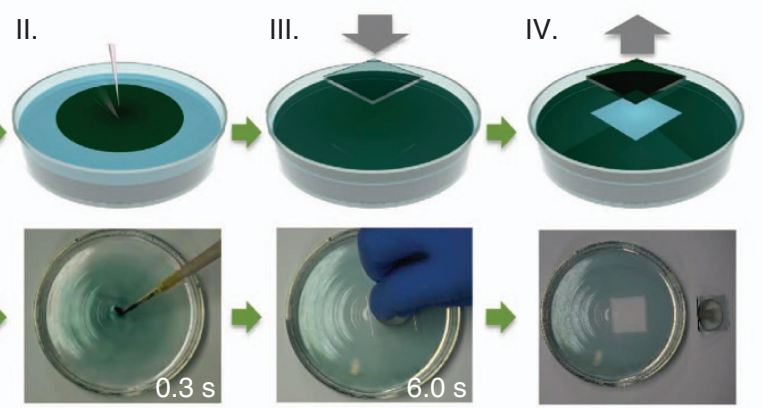

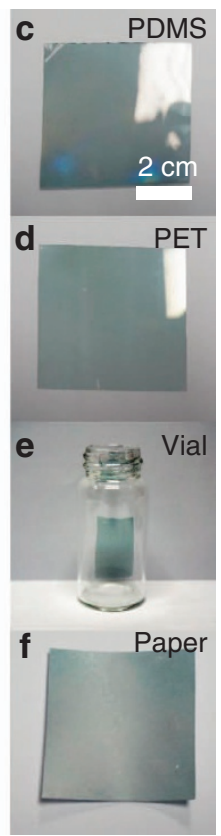

Figure 1 | Principle and formation process of spontaneous spreading. (a) Spreading of polymer solution dropped onto the base solution is determined by the spreading coefficient, S. Positive $S$ results in a uniform polymer film; otherwise, polymer drops aggregate; (b) Schematic illustrations of formation of an SS BHJ film on water and transfer to target substrates. Actual processing images are shown. (c-f) Transferred SS-PTB7:PC 71 BM films on various substrates: PDMS (c), PET (d), copy paper (e) and a curved surface of a vial (f).

appeared to be uninfluenced by the presence of $\mathrm{PC}_{71} \mathrm{BM}$ and an additive such as DIO.

High-resolution transmission electron microscope analysis of the BHJ films was performed under the same defocused imaging conditions $(10 \mu \mathrm{m})$ to compare the domain sizes of PTB7 (refs 24,25). To fabricate top-down transmission electron microscope (TEM) samples, the SS-PTB7:PC ${ }_{71} \mathrm{BM}$ film was directly transferred to 300 mesh copper TEM grids, while the spin-coated (SC-)PTB7:PC $\mathrm{P}_{71} \mathrm{BM}$ films (air and $\mathrm{N}_{2}$ ) were floated on deionized water and transferred. To highlight the $\mathrm{PC}_{71} \mathrm{BM}$ cluster domains (dark regions) and PTB7 crystallite domains (bright regions), the bright regions are coloured red (Supplementary Note 5; Supplementary Fig. $6 \mathrm{~d}-\mathrm{f}$ ). The $\mathrm{PC}_{71} \mathrm{BM}$ cluster domains and domains of PTB7 crystallites in the SC-PTB7:PC ${ }_{71} \mathrm{BM}$ films processed in air and in $\mathrm{N}_{2}$ were slightly larger than those of the SS-PTB7:PC ${ }_{71} \mathrm{BM}$ film (Fig. $\left.2 \mathrm{a}-\mathrm{c}\right)^{26}$. Figure $2 \mathrm{c}$ illustrates the more uniformly formed nanomorphology of the SS-PTB7:PC ${ }_{71} \mathrm{BM}$ film with finely dispersed phase separation between the PTB7 and $\mathrm{PC}_{71} \mathrm{BM}$ domains, suggesting efficient spatial interaction between these species during the SS process. The size distribution of the PTB7 domains was determined from at least five samples using ImageJ software (Fig. 2d; see Supplementary Note 6 and Supplementary Fig. 7 for details). The average size of the PTB7 domains in the SSPTB7:PC ${ }_{71} \mathrm{BM}$ film was $5.50 \mathrm{~nm}$, while that of the SC-PTB7:PC ${ }_{71} \mathrm{BM}$ films was $8.79 \mathrm{~nm}$ on average; the PTB7 domains in the SS film had a narrower size distribution.

The final morphology of a BHJ film with solvent additives is governed by the dynamics of solvent evaporation and liquidliquid phase separation ${ }^{26,27}$. Immediately on dropping the solution on the water, the solvent (that is, $\mathrm{CB}$ ) volume decreases due to evaporation and dissolution of the solvent into the water (Fig. 1a); the PTB7 chains start to form aggregated fine structures within a few seconds. This is much faster than the drying of spin-coated BHJ films (a few tens of seconds) ${ }^{28}$.

However, too rapid solvent evaporation and a higher initial concentration of the polymer in the solution at the air/liquid interface may enhance the nucleation rate, leading to a high number of small crystallites ${ }^{29,30}$. Therefore, inducing optimizedscale phase separation with appropriately sized crystallites is desirable. To properly control the phase separation, the DIO content in the PTB7:PC $\mathrm{P}_{71} \mathrm{BM}$ solution was varied ${ }^{26}$. Because DIO has a higher boiling point $\left(\sim 170^{\circ} \mathrm{C}\right)$ and higher surface tension (43.25 dynes $\mathrm{cm}^{-1}$ ) than $\mathrm{CB}$, mixing 10 vol\% DIO with PTB7:PC ${ }_{71} \mathrm{BM}$ solution leads to increased surface tension of the droplet, which slows the rate of spreading over the water ${ }^{31,32}$. Furthermore, DIO molecules with greater specific gravity $\left(1.84 \mathrm{~g} \mathrm{~cm}^{-3}\right)$ than water are steadily removed from films into water during SS process although DIO is insoluble in water (water solubility at $25^{\circ} \mathrm{C}: 0.2301 \mathrm{mgl}^{-1}$ ). This results in sufficient plasticity to allow the aggregated polymer chains to be mobile enough to assume the optimal conformations ${ }^{31,33}$. At the same time, the $\mathrm{PC}_{71} \mathrm{BM}$ molecules remain dissolved in DIO, preventing large-scale liquid-liquid phase separation ${ }^{34}$. Subsequently, the $\mathrm{PC}_{71} \mathrm{BM}$ molecules captured in DIO are slowly integrated into the PTB7 crystallites before complete solidification, resulting in larger interface areas between smaller PTB7 and $\mathrm{PC}_{71} \mathrm{BM}$ domains ${ }^{26}$. The larger interfacial areas between domains result in efficient exciton dissociation in the BHJ PSCs, as discussed below ${ }^{35}$.

Figure $2 \mathrm{e}$ shows photoluminescence spectra (excited at $633 \mathrm{~nm}$ ) of the SC- and SS-PTB7:PC ${ }_{71}$ BM films on silicon wafers. Significant quenching of the photoluminescence intensity for the SS-PTB7:PC ${ }_{71}$ BM film confirms facilitated exciton dissociation in the increased interfacial area between the finely dispersed phaseseparated domains, as revealed in the TEM images (Fig. 2c).

The influence of the SS process on the degree of molecular order was probed using resonant Raman spectroscopy (excited at $633 \mathrm{~nm}$ ) of the SC- and SS-PTB7:PC ${ }_{71} B M$ films because these spectra can reveal the intramolecular conformational order of polymer chains (Fig. $2 \mathrm{~g})^{36,37}$. The wavelength of $633 \mathrm{~nm}$ is close to the maximum absorption of PTB7 (Fig. 2h), which allows excitation of even the disordered phase of PTB7 in strong resonance with electronic transitions ${ }^{37}$. The Raman spectra were background corrected and the spectra from three different regions of the samples were averaged. Spectra were also acquired from 
a

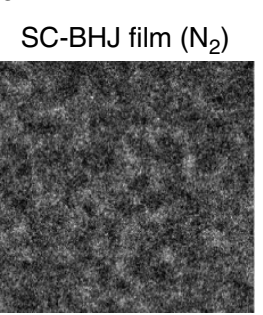

b

SC-BHJ film (air)

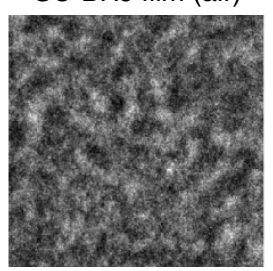

C

SS-BHJ film (air)

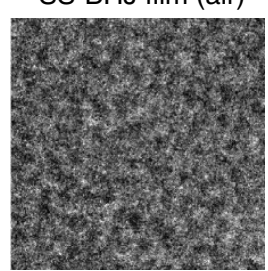

d

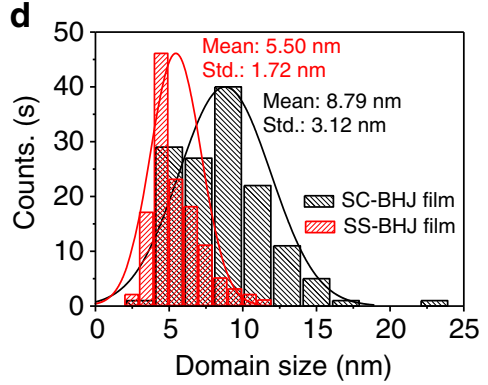

e

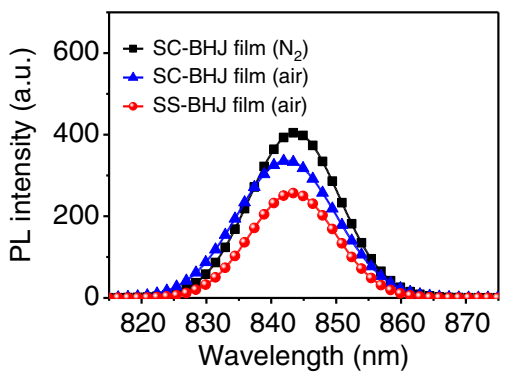

f

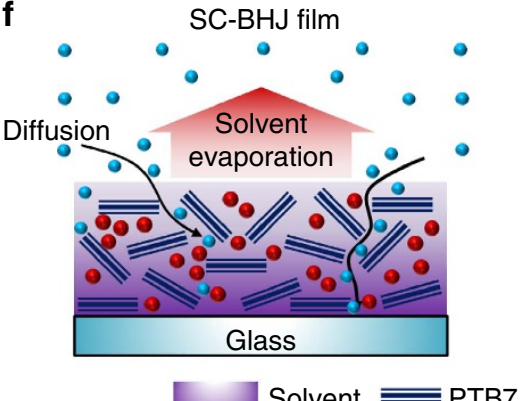

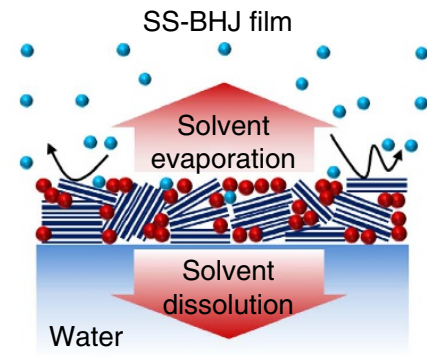

- $\mathrm{PC}_{70} \mathrm{BM} \cdot \mathrm{O}_{2}$

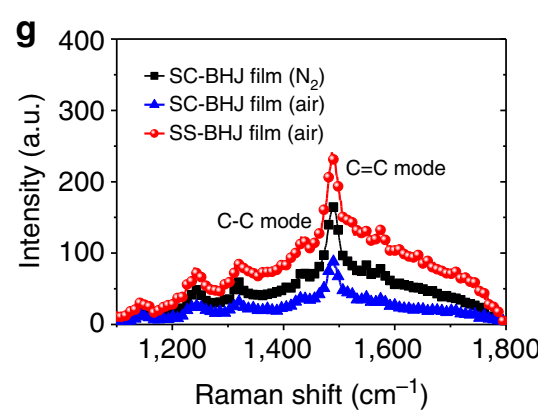

j

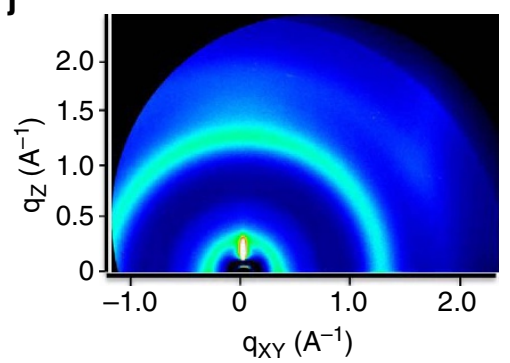

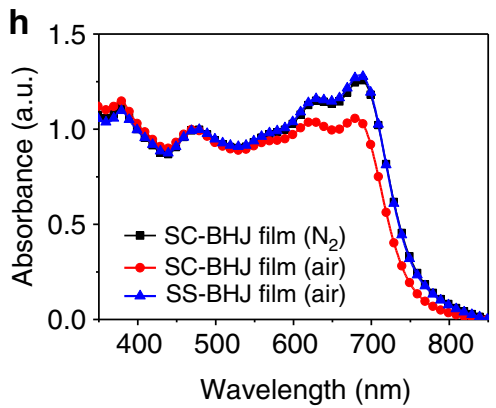

$\mathbf{k}$

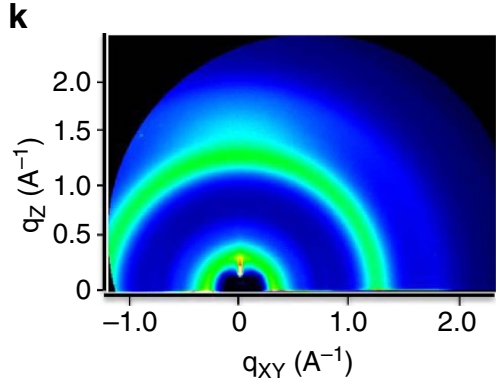

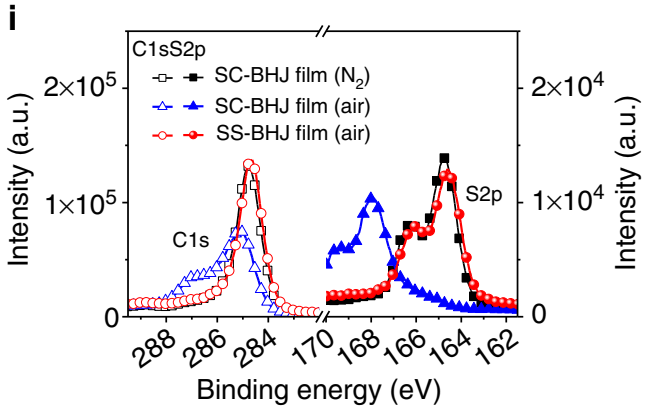

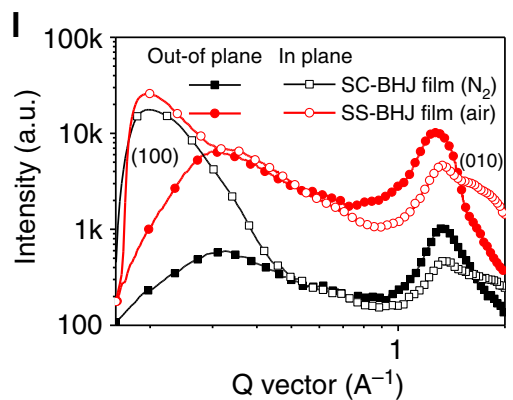

Figure 2 | High-quality polymer from spontaneous spreading process. (a-c) TEM images of $\mathrm{N}_{2}$ processed (a) air-processed SC-BHJ (b) and SS-BHJ (c) films measured at the same defocusing distance. (d) PTB7 domain size distribution histograms of $\mathrm{N}_{2}$-processed SC-BHJ and SS- BHJ films. (e) Photoluminescence spectra of both films when excited at $633 \mathrm{~nm}$. (f) SC-BHJ and SS-BHJ films immediately after film formation process. (g-i) Raman spectra ( $\mathbf{g}$ ) and ultraviolet-visible absorption spectra (h) for SC-BHJ and SS-BHJ films, and S2p and C1s XPS spectra (i) of $\mathrm{N}_{2}$ and air-processed SC-BHJ, and SS- BHJ films. (j-I) 2D GIWAXS images of SC-BHJ (j) and SS-BHJ films (k) and out-of-plane and in plane GIWAXS profiles (I).

three samples prepared at different times to exclude sample-to sample variation and measurement error. The Raman peaks of the SS-PTB7:PC ${ }_{71} \mathrm{BM}$ film at $\sim 1435 \mathrm{~cm}^{-1}$ (C-C intra-ring stretch mode) and at $\sim 1,490 \mathrm{~cm}^{-1}$ (symmetric $\mathrm{C}=\mathrm{C}$ stretch mode) were almost identical to those of the SC-films. The ratio of the intensity of the peaks of the $\mathrm{C}-\mathrm{C}$ and $\mathrm{C}=\mathrm{C}$ modes $\left(I_{\mathrm{C}-\mathrm{C}} / I_{\mathrm{C}=\mathrm{C}}\right.$ of the SS film) increased from 0.46 to 0.49 , and the full width at half maximum (FWHM) of the $\mathrm{C}=\mathrm{C}$ mode became narrower, reflecting the enhanced degree of molecular order of the PTB7 chains in the SS film.

Grazing-incidence wide-angle X-ray scattering (GIWAXS) was also used to verify enhanced crystallization and orientation of the molecules during the spontaneous spreading process. The intensity of the (010) scattering peak at $1.6 \AA^{-1}$ for the SS-PTB7:PC ${ }_{71} \mathrm{BM}$ film increased markedly compared with that of the SC-PTB7:PC ${ }_{71} B M$ film (Fig. $2 \mathrm{j}-1$ ), indicating enhanced face-on orientation of the PTB7 chains in relation to the substrate in the SS-PTB7:PC ${ }_{71} \mathrm{BM}$ film processed in air. The enhanced faceon orientation of the SS-PTB7 film is most likely due to the interplay of Van der Waals interaction from alkyl side chains and the attractive interactions occurring between the less hydrophobic backbone of PTB7 and the water surface ${ }^{38,39}$. The intensity ratio $\left(I_{\text {out }} / I_{\text {in }}\right)$ of the out-of-plane to in-plane PTB7 lamellar peaks (100) at around $0.35 \AA^{-1}$ was also calculated for quantitative 
analysis of the edge-on PTB7 lamellar to face-on crystallite ratio ${ }^{40}$. The $I_{\text {out }} / I_{\text {in }}$ ratio of the SS-PTB7:PC ${ }_{71} \mathrm{BM}$ film was 3.96, whereas that of the SC-PTB7:PC ${ }_{71} \mathrm{BM}$ film was 29.1, indicating a significantly lower degree of edge-on orientation.

Enhanced crystallization and orientation of the molecules in the SS-PTB7:PC ${ }_{71} B M$ film can affect the air stability of the film ${ }^{41-43}$. The mass density of an organic thin film, which is determined by the degree of crystallinity, can alter the ability of chemical reactants to permeate to the reaction site of molecules ${ }^{41}$. Enhanced molecular packing can also strengthen chemical bonds in the film due to more rigid and planar backbones, extending the regions of delocalization, leading to a chemically more robust film $^{42,43}$.(Supplementary Note 7; Supplementary Fig. 8)

Air processability. The air stability of the films formed by various processes was evaluated based on the Raman spectra of the SC- and SS-PTB7:PC ${ }_{71} \mathrm{BM}$ films obtained under $514 \mathrm{~nm}$ excitation. Raman peaks corresponding to phenylene-alkoxy (-R-O-) stretching $\left(\sim 1,250\right.$ and $\left.\sim 1,350 \mathrm{~cm}^{-1}\right)$ and the $\mathrm{C}=\mathrm{C}$ stretching mode of the BDT group $\left(\sim 1,500, \sim 1,550 \mathrm{~cm}^{-1}\right.$ and $\sim 1,580 \mathrm{~cm}^{-1}$ ) were observed for the air-processed SCPTB7:PC ${ }_{71} B M$ film (Supplementary Note 8; Supplementary Fig. 9). The intensity of these peaks was significantly lower than that of the peaks of the $\mathrm{N}_{2}$-processed SC-PTB7:PC ${ }_{71} \mathrm{BM}$ film. This suggests that oxygen that infiltrated into the film during the spin-coating and drying processes contributes to cleavage of the alkoxy groups and insertion of oxygen into the $\mathrm{C}=\mathrm{C}$ bonds in the backbone, resulting in disruption of the $\pi$-conjugation. This is consistent with the decreased and slightly blue-shifted absorption and photoluminescence of the air-processed SC-PTB7:PC ${ }_{71} B M$ film (Fig. 2h,e).

A survey scan using X-ray photoelectron spectroscopy (XPS) was also performed to confirm the air stability of the SS films ${ }^{44,45}$. Figure 2i shows a considerable shift and peak broadening of the S2p peak for the air-processed SC-PTB7:PC ${ }_{71} B M$ film, which can be ascribed to chain scissions in the polymer backbone, or to loss of the side chains. The $\mathrm{C} 1 \mathrm{~s}$ spectrum of the air-processed SC-PTB7:PC ${ }_{71} B M$ film shows the emergence of a peak at $287 \mathrm{eV}$, derived from oxygenated carbon species, including a carboxyl group $^{13}$. In contrast, no detectable S2p peak broadening or C1s peak from oxygenated carbon species was observed for the SS-BHJ and $\mathrm{N}_{2}$-processed SC-BHJ films (Fig. 2i).

Surprisingly, no indication of degradation was observed for the SS-PTB7:PC 71 BM films based on the XPS and Raman spectra (Fig. 2i; Supplementary Fig. 9) although the film was formed on water in air. For the air-processed SC-BHJ films, CB remaining between the polymer chains before complete drying may work as effective percolation pathways for diffusion of oxygen to the PTB7 molecules because of the significantly higher oxygen diffusivity of $\mathrm{CB}\left(1.16 \times 10^{-5} \mathrm{~cm}^{2} \mathrm{~s}^{-1}\right)$ than that of PTB7 $\left(1.2 \times 10^{-8} \mathrm{~cm}^{2} \mathrm{~s}^{-1}\right)^{46,47}$, which can facilitate reactions between

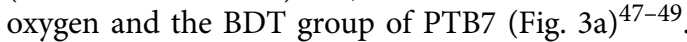

To examine the aftermath effects of remaining solvent on degradation of the $\mathrm{BHJ}$ films, the atomic ratio of oxygen to sulfur in the polymer films exposed to ambient conditions versus the drying time was probed by using XPS. Briefly, the $\mathrm{BHJ}$ films were spin-coated under $\mathrm{N}_{2}$ and subsequently exposed to air for different periods; finally, the films were completely dried in a $\mathrm{N}_{2}$-filled glove box (Supplementary Note 9; Supplementary Fig. 10a). The films exposed to air for a longer time exhibited greater oxygen permeation with a broad distribution of oxygen in the films, implying that the $\mathrm{CB}$ molecules could provide pathways for oxygen diffusion into the BHJ film, as presented in Fig. 3a. In contrast, the $\mathrm{O} / \mathrm{S}$ ratio and the atomic distribution of oxygen in SS-PTB7:PC $C_{71} B M$ film were comparable to that of the
$\mathrm{N}_{2}$-processed SC-PTB7: $\mathrm{PC}_{71} \mathrm{BM}$ film, suggesting excellent air processability by the spontaneous spreading process (Supplementary Note 9; Supplementary Fig. 10b).

The effect of solvent (here, $\mathrm{CB}$ ) on oxidation of polymer films was evaluated. DIO additive as a co-solvent was not considered because DIO molecules are steadily removed from films during the SS process due to greater specific gravity of DIO than water, as previously mentioned.(Supplementary Table 1, Supplementary Note 10 and Supplementary Fig. 11) For the experiments, PTB7 only (that is, without fullerenes) films were prepared using different post-treatments. PTB7 films spun in nitrogen were treated under different conditions (Fig. 3a) before exposure to air for $3 \mathrm{~h}$, that is, (i) as-spun without removal of solvent, (ii) thermally treated at $70^{\circ} \mathrm{C}$ for $20 \mathrm{~min}$. After exposure to air for $3 \mathrm{~h}$, the intensity of the absorption peaks of the as-spun PTB7 film with excess solvent were considerably reduced (Fig. 3b), indicating degradation of the PTB7 polymer chains due to air exposure, as compared with the thermally treated PTB7 films. Moreover, the Raman peaks of the as-spun PTB7 film obtained under excitation at $514 \mathrm{~nm}$ were also significantly diminished relative to those of the other films, suggesting cleavage of the alkoxy groups and insertion of oxygen into the $\mathrm{C}=\mathrm{C}$ bonds in the backbone (Fig. 3c). Oxygen within the three PTB7 films was tracked using time-of-flight secondary ion mass spectrometry (TOF-SIMS), as shown in the depth profile in Fig. 3d. Figure 3e-g shows the $3 \mathrm{D}$ iso-surface overlay of the intensity of the oxygen ion peaks $\left({ }^{16} \mathrm{O}\right.$ and $\left.{ }^{18} \mathrm{O}\right)$. The intensity of oxygen near the surface of the as-spun PTB7 film was considerably higher than that of the thermally-treated PTB7 film (Fig. 3e-f), confirming that oxygen molecules penetrated the PTB7 film via the excess solvent between the PTB7 chains as explained above. In contrast, the oxygen intensity near the surface region of the SS-PTB7 film was comparable to that of the thermally treated film (Fig. 3f,g). In addition, performed studies on the effect of solvent on exposure to light and air on the oxidation of PTB7 films also suggest that oxygen adsorbed through the solvent path in the film during illumination in air contributes to accelerated disruption of the backbone conjugation of BDT group in PTB7 molecules. (Supplementary Note 11; Supplementary Fig. 12)

It can be inferred that the spontaneous spreading process can induce rapid formation of a condensed and well-packed thin film due to the ultrafast solvent removal, which can prevent the intrusion of oxygen via the $\mathrm{CB}$ solvent, impeding oxidation and redistribution of the polymer and $\mathrm{PC}_{71} \mathrm{BM}$ molecules in the $\mathrm{BHJ}$ film.

Photovoltaic performances. Polymer solar cells (hereafter, SS-PSCs) were fabricated to evaluate the applicability of the SS films to high-efficiency functional devices fabricated in air. For comparison with the SS-PSCs, SC-PSCs were fabricated under two different sets of environmental conditions, that is, in a $\mathrm{N}_{2}$ filled glove box (hereafter, SC-PSCs $\left(\mathrm{N}_{2}\right)$ ) and under ambient atmosphere (hereafter, SC-PSCs (air)). The air exposure time of the SC-PSCs (air) and SS-PSCs during the coating process in air was set to under $10 \mathrm{~min}$. The post-drying process for the SC-PSCs (air and $\mathrm{N}_{2}$ ) was performed in a $\mathrm{N}_{2}$ filled glove box (see Methods section). The device structure (Fig. 4a,e) was ITO/CBA (or $\mathrm{ZnO}$ )/ PTB7 (or PTB7-Th): $\mathrm{PC}_{71} \mathrm{BM} / \mathrm{BCP} / \mathrm{Al}$ (or $\mathrm{MoO}_{3} / \mathrm{Ag}$ ).

Figure $4 \mathrm{~b}$,f show the representative current density-voltage $(J-V)$ characteristics of the SS-PSCs, SC-PSCs $\left(\mathrm{N}_{2}\right)$ and SC-PSCs (air) based on a mixture of PTB7:PC ${ }_{71} \mathrm{BM}$ and PTB7-Th:PC $\mathrm{P}_{71} \mathrm{BM}$. The SC-PSCs (air) exhibited much lower performance than the SS-PSCs and SC-PSCs $\left(\mathrm{N}_{2}\right)$, attributed to degradation of the air-processed $\mathrm{BHJ}$ films by oxidization as explained in relation to Fig. $2 f$. 
a

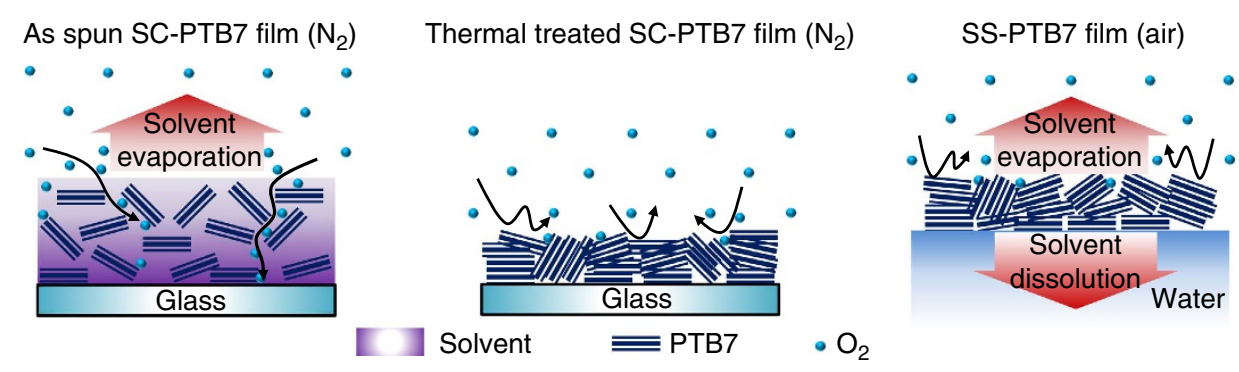

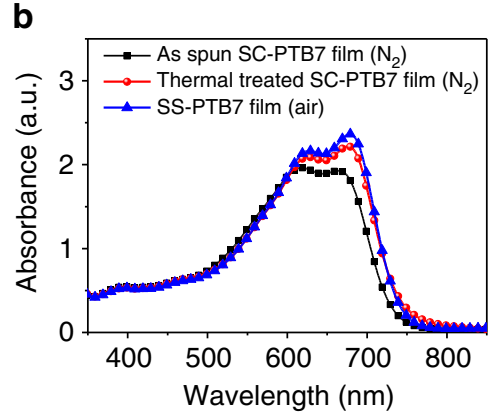

b

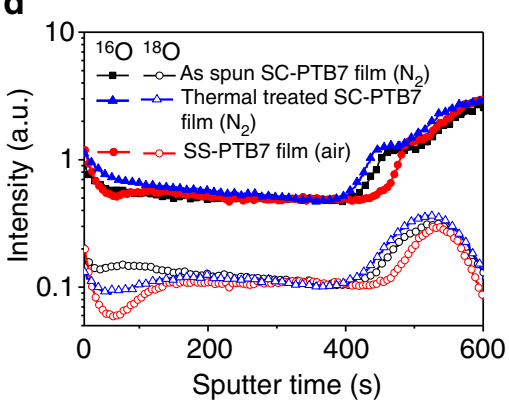

C

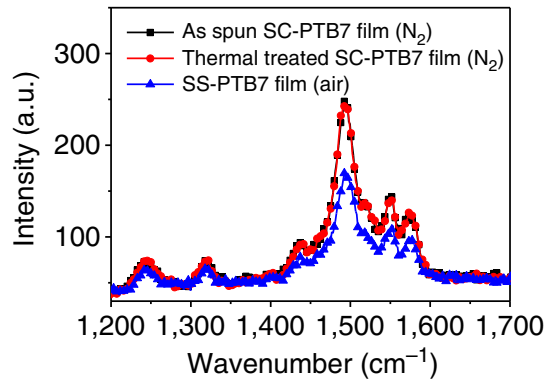

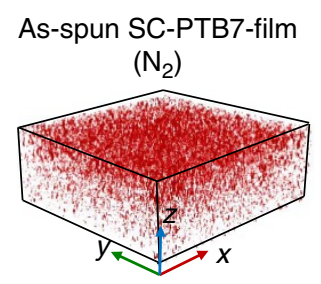

f

g

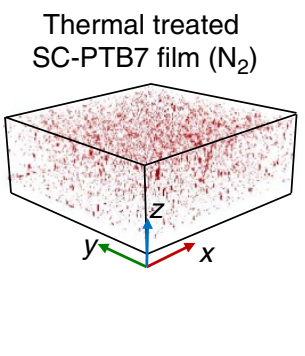

SS-PTB7 film (air)

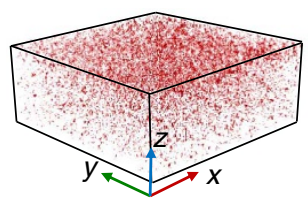

Figure 3 | Oxygen diffusion into polymer films via solvent. (a) Oxygen diffusion mechanism in PTB7 films during drying process for spin-coated PTB7 films treated under different conditions and for SS-PTB7 film. (b) Ultraviolet-visible absorption spectra and (c) Raman spectra of spin-coated PTB7 films treated under different conditions, ( $\mathbf{d}-\mathbf{g}$ ) TOF-SIMS depth profile and 3D iso-surface overlay of oxygen ions $\left({ }^{16} \mathrm{O}\right.$ and $\left.{ }^{18} \mathrm{O}\right)$ intensity as a function of sputter time for the spin-coated PTB7 films treated under different conditions.

The PCE of the PTB7-based SS-PSC was 6.27\% (Table 1, Supplementary Note 12 and Supplementary Table 2), which is comparable to that of the SC-PSC $\left(\mathrm{N}_{2} ; 6.21 \%\right)$. However, the PCE of the SC-PSCs (air) was significantly reduced (3.74\%). The PCE of the PTB7-Th-based SS-PSC reached $8.44 \%$, and this is the highest PCE reported for air-processed organic solar cells to date to the best of our knowledge. Details of the device performance are summarized in Table 1.

Both polymer-based SS-PSCs exhibited performance comparable to that of the SC-PSCs $\left(\mathrm{N}_{2}\right)$ with an almost identical spectral response in the external quantum efficiency (EQE) data (Fig. 4c,g). To evaluate the reliability of the SS-PSCs processed in air, statistical data for the PCEs of 30 PTB7 and PTB7-Th devices are presented in Fig. 4d,h and Supplementary Figs 13 and 14 with Supplementary Note 13, respectively.

We demonstrated the potential of our method as a scalable process by transferring the SS film to a large PET substrate using an R2R process. Figure $4 \mathrm{i}$ shows a SS -PTB7:PC ${ }_{71} \mathrm{BM}$ film on the water bath $\left(30 \times 120 \mathrm{~cm}^{2}\right.$ size $)$, made by dropping only $200 \mu \mathrm{l}$ of the polymer solution as a point source for spontaneous spreading (Supplementary Movie 2). After the solution spread, a $1 \mathrm{~m}$ long SS film was obtained in a few seconds. A custom-made R2R system was used to transfer the large SS film to the PET substrate (Fig. 4j). The SS-film was transferred successfully from the water surface to the PET substrate (Supplementary Movie 3). Figure 4k shows a PET film coated with a PTB7:PC ${ }_{71}$ BM layer $\sim 60 \mathrm{~nm}$ thick. We anticipate that this new polymer film formation method will have great potential as a new process for R2Rprocessed modules with low material consumption and drying time, and consequently lower fabrication cost.

In summary, we developed an air-processable technique to form polymer films on an aqueous surface within a few seconds, using the spontaneous spreading phenomenon. Ultrafast removal of solvent during the process causes the films to have uniform and high-quality nanomorphology because oxygen diffuses into the polymer films rapidly through the remaining solvent between the polymer chains. Furthermore, the polymer films were successfully transferred onto various substrates. Finally, we demonstrated high performance of PSCs prepared using the proposed process, comparable to that of PSCs prepared by spin coating. We expect that this approach can be extended by roll-to-roll production to achieve high-quality films.

\section{Methods}

Preparation of the polymer solution. For the SC films, PTB7:PC ${ }_{71} \mathrm{BM}$ (1-Materials: Nano-C) and PTB7-Th:PC ${ }_{71}$ BM (1-Materials: Nano-C) at a weight ratio of 1:1.5 were dissolved in chlorobenzene $(\mathrm{CB}): 1,8$-diiodoctane (DIO) $(97: 3 \mathrm{v} / \mathrm{v}$ ) at $50^{\circ} \mathrm{C}$. This mixture was stirred for $12 \mathrm{~h}$ under $\mathrm{N}_{2}$. For the SS-films, PTB7:PC $\mathrm{P}_{71} \mathrm{BM}$ 
a

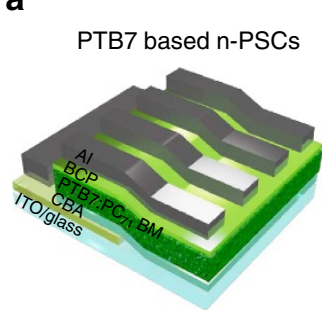

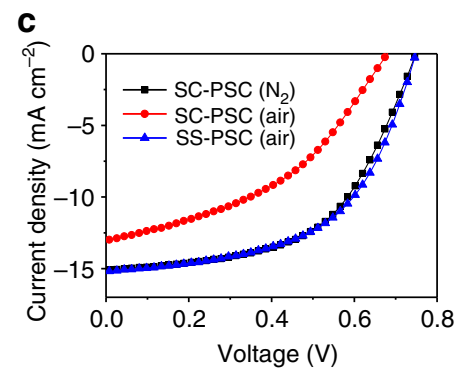

b

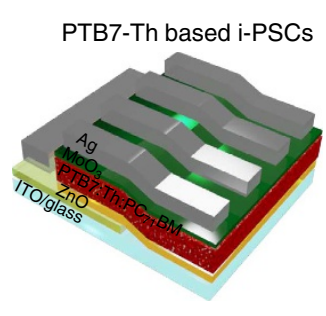

d

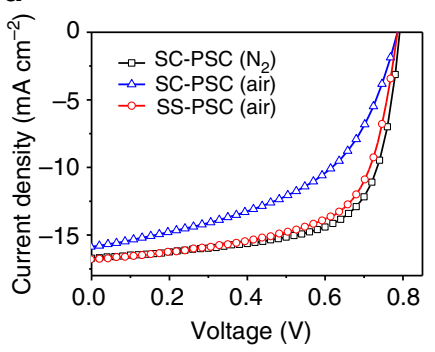

e

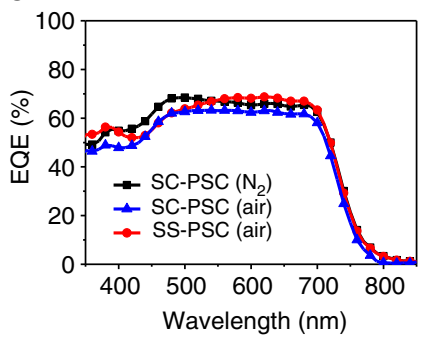

f

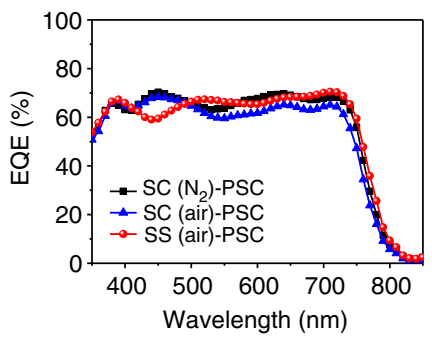

g

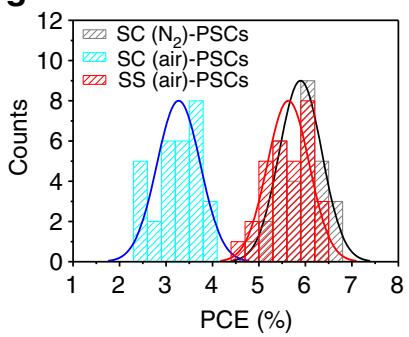

h

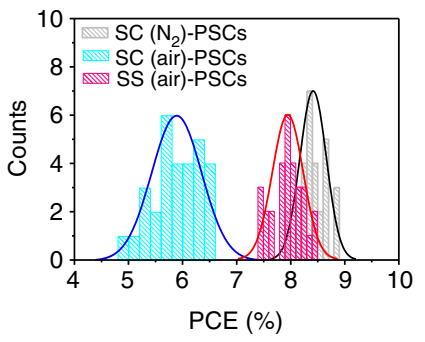

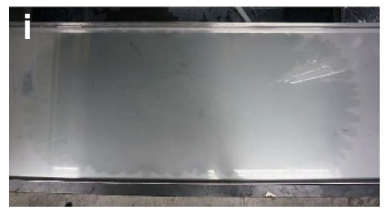
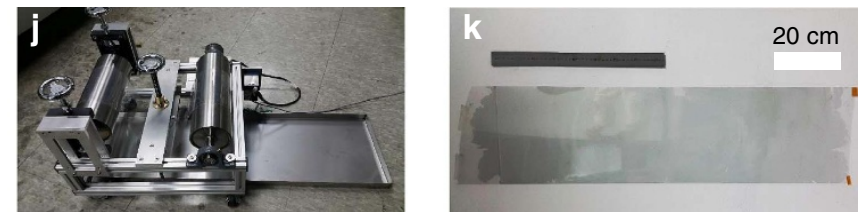

Figure 4 | Fabrication of organic solar cells using SS films and potential for scalable process. $(\mathbf{a}, \mathbf{b})$ Device structure of $n-P S C\left(P T B 7: P C{ }_{71} B M\right)$ and $i-P S C$ (PTB7-Th:PC 71 BM); (c-f) J-V characteristics and EQE spectra of PTB7-based (c,e) and PTB7-based PSCs (d,f) under AM $1.5 \mathrm{G} 100 \mathrm{~mW} \mathrm{~cm}^{-2}$ illumination. $(\mathbf{g}, \mathbf{h})$ statistics for PCEs of 30 samples. (i) Formation of a single SS film on a large water bath $\left(30 \times 120 \mathrm{~cm}^{2}\right)$. (j) Homemade R2R coating system for largescale transfer on flexible substrates. (k) $25 \times 100 \mathrm{~cm}^{2}$ PET film coated with a SS-PTB7:PC 71 BM film.

Table 1 | Representative photovoltaic performance parameters for SC- and SS-PSCs.

\begin{tabular}{|c|c|c|c|c|}
\hline PSCs & Performance & SC-PSC $\left(\mathbf{N}_{\mathbf{2}}\right)$ & SC-PSC (air) & SS-PSC (air) \\
\hline PTB7:PC ${ }_{71} B M$ & $\begin{array}{c}\text { PCE (\%) } \\
J_{\mathrm{sc}}(\mathrm{mAcm}-2) \\
V_{\mathrm{oc}}(\mathrm{V}) \\
\mathrm{FF}\end{array}$ & $\begin{array}{r}5.90 \pm 0.27(6.34) \\
14.22 \pm 0.83(15.71) \\
0.74 \pm 0.03(0.77) \\
0.56 \pm 0.03(0.60)\end{array}$ & $\begin{array}{c}3.32 \pm 0.25(3.74) \\
12.27 \pm 0.67(13.28) \\
0.65 \pm 0.03(0.69) \\
0.42 \pm 0.02(0.46)\end{array}$ & $\begin{array}{c}5.84 \pm 0.25(6.24) \\
14.71 \pm 0.57(15.45) \\
0.74 \pm 0.01(0.76) \\
0.54 \pm 0.02(0.57)\end{array}$ \\
\hline PTB7-Th:PC ${ }_{71} \mathrm{BM}$ & $\begin{array}{c}\text { PCE (\%) } \\
J_{\mathrm{sc}}\left(\mathrm{mAcm} \mathrm{cm}^{-2}\right) \\
V_{\mathrm{oc}}(\mathrm{V}) \\
\mathrm{FF}\end{array}$ & $\begin{array}{c}8.41 \pm 0.24(8.86) \\
16.80 \pm 0.27(16.72) \\
0.80 \pm 0.01(0.79) \\
0.63 \pm 0.02(0.67)\end{array}$ & $\begin{array}{c}5.89 \pm 0.46(6.29) \\
14.71 \pm 0.84(15.85) \\
0.78 \pm 0.01(0.79) \\
0.51 \pm 0.03(0.50)\end{array}$ & $\begin{aligned} 7.95 & \pm 0.28(8.44) \\
16.66 & \pm 0.34(16.80) \\
0.79 & \pm 0.01(0.79) \\
0.60 & \pm 0.02(0.64)\end{aligned}$ \\
\hline
\end{tabular}

PSCs, polymer solar cells; PTB7, poly[[4,8-bis[(2-ethylhexyl)oxy]benzo[1,2-b:4,5-b']dithiophene-2,6-diyl][3-fluoro-2-[(2-ethylhexyl) carbonyl]thieno[3,4-b]thiophenediyl]]; SS, spontaneously spreading.

Means and s.d. are obtained from 30 devices. The values in brackets illustrates the best cell performance for each device.

and PTB7-Th:PC $\mathrm{C}_{71} \mathrm{BM}$ at a weight ratio of 1:1.5 were dissolved in $10 \mathrm{vol} \%$ of DIO in $\mathrm{CB}(\mathrm{CB}: \mathrm{DIO}=90: 10 \mathrm{v} / \mathrm{v})$.

Solar cell fabrication for PTB7:PC ${ }_{71}$ BM. PTB7:PC ${ }_{71}$ BM PSCs were fabricated on ITO-deposited glass substrates $\left(R_{\mathrm{sh}}: 22 \mathrm{ohm} \mathrm{sq}{ }^{-1}\right)$. A layer of 4-chlorobenzoic acid (CBA, Sigma-Aldrich) as a hole transfer layer (HTL) was spun onto the substrates at 3,000 r.p.m. for $30 \mathrm{~s}$; the substrates were then annealed at $70^{\circ} \mathrm{C}$ for $5 \mathrm{~min}$ (ref. 50). Surplus molecules of CBA on the substrates were removed by spin coating with methanol at 6,000 r.p.m. for 30 s. Two layers of SS-PTB7: $\mathrm{PC}_{71} \mathrm{BM}$ films were transferred sequentially onto the substrates and dried at $70^{\circ} \mathrm{C}$ for 10 min under $\mathrm{N}_{2}$. SC-PTB7: $\mathrm{PC}_{71} \mathrm{BM}$ films were spun at 2,500 r.p.m. for $30 \mathrm{~s}$ and then dried at $70^{\circ} \mathrm{C}$ for 10 min under $\mathrm{N}_{2}$. Subsequently, bathrocuproine $(\mathrm{BCP}, 8 \mathrm{~nm})$ and $\mathrm{Al}(150 \mathrm{~nm})$ were deposited through a shadow mask by thermal evaporation onto the devices ${ }^{51}$.

Solar cell fabrication for PTB7-Th:PC ${ }_{71}$ BM. PTB7-Th:PC ${ }_{71}$ BM PSCs were fabricated on the same ITO glass substrates with PTB7:PC $\mathrm{P}_{71} \mathrm{BM}$ PSCs. A layer of
$\mathrm{ZnO}$ for electron transfer layer (ETL) was spun onto the substrates at 6,000 r.p.m. for $30 \mathrm{~s}$, and the substrates were then annealed at $200^{\circ} \mathrm{C}$ for $20 \mathrm{~min}$ (ref. 25). Two layers of SS-PTB7:PC ${ }_{71} \mathrm{BM}$ films were transferred sequentially onto the substrates. The SC-PTB7: $\mathrm{PC}_{71} \mathrm{BM}$ films were spun at 2,000 r.p.m. for $30 \mathrm{~s}$. There was no additional drying process or annealing. Subsequently, $\mathrm{MoO}_{3}(9 \mathrm{~nm})$ and $\mathrm{Ag}$ $(150 \mathrm{~nm})$ were deposited through a shadow mask by thermal evaporation onto the devices $^{52}$.

Measurement of polymer solar cells. The current density-voltage $(J-V)$ characteristics of the cells were measured from 1.0 to $-0.2 \mathrm{~V}$ using a solar simulator (K201 LAB55, McScience) under irradiance of $100 \mathrm{~mW} \mathrm{~cm}^{-2}$ from a $150 \mathrm{~W}$ Xe short-arc lamp filtered by an air mass $1.5 \mathrm{G}$ filtre. Light intensity was calibrated with a Si reference cell (McScience, K801S-K302). The voltage scan rate was $100 \mathrm{mV} \mathrm{s}^{-1}$ and no device preconditioning was applied before starting the measurement, such as light soaking. A $6.25 \mathrm{~mm}^{2}$ aperture mask was attached to the device to define the illuminated area clearly. The quantum efficiency $(\mathrm{QE})$ spectra of the solar cells were obtained using a spectral (K3100 IQX, McScience Inc.) 
measurement system. The integrated response $\left(J_{S C}\right)$ under the standard reference spectrum was compared with the $J_{S C}$ value measured under the simulator, and they showed an $\sim 2 \% J_{S C}$ difference.

Film analysis. The surface morphology of the $\mathrm{BHJ}$ films was observed using atomic force microscopy in tapping mode under ambient conditions (AFM, Nanoman, Veeco) using a field emission scanning electron microscope (FE-SEM, FEI Sirion) and a field emission transmission electron microscope (FE-TEM, FEI Tecnai G2 f30 S-Twin, $300 \mathrm{KeV}$ ). Photoluminescence quenching was evaluated for the SC- and SS-PTB7:PC ${ }_{71} \mathrm{BM}$ films without metal electrodes using a Horiba Jobin Yvon NanoLog spectrophotometer. We performed the measurement with at least three samples to reduce the sample-to-sample variation and measurement error. The XPS and ultraviolet photoelectron spectroscopy spectra were recorded with a Sigma Probe (Thermo VG Scientific) instrument, using a micro-focused monochromatic $\mathrm{Al} \mathrm{X}$-ray source with a beam energy of $1468.74 \mathrm{eV}$ and a takeoff angle of $45^{\circ}$. Raman spectra were obtained using a micro-Raman system (LabRam HR spectrometer, JY Horiba). A $514 \mathrm{~nm}$ solid-state laser and a $633 \mathrm{~nm} \mathrm{He}-\mathrm{Ne}$ laser were used as the excitation light sources and were kept to less than $0.5 \mathrm{~mW}$ to prevent photo-degradation of the $\mathrm{BHJ}$ films. The laser was focused to a beam diameter of $\sim 5 \mu \mathrm{m}$ on the sample using a $\times 50$ objective microscope lens. The Raman spectra were background corrected and obtained by averaging the spectra obtained from three different regions of each sample. The spectra from the different regions were almost the same, demonstrating the reliability of the results. We also confirmed the outcomes by measuring three samples prepared at different times. The spectral resolutions are within 0.4 and $0.2 \mathrm{~cm}^{-1}$ for the 514 and $633 \mathrm{~nm}$ excitations, respectively. GIWAXS measurement was performed using X-rays with a wavelength of $\lambda=1.54 \AA$ at the $8 \mathrm{C} 1$ beam line of the Pohang Accelerator Laboratory. The $3 \mathrm{D}$ profiles for oxygen distribution in the PTB7 films were obtained using a TOF-SIMS 5 system (ION-TOF GmbH, Münster, Germany) equipped with a $30 \mathrm{KeV}$ liquid metal ion gun and a time-of-flight mass spectrometer. To sputter the PTB7 film, a $1 \mathrm{KeV}$ beam of $\mathrm{Cs}^{+}$ions was used to remove material by sputtering a $300 \mu \mathrm{m} \times 300 \mu \mathrm{m}$ region, whereas a $30 \mathrm{KeV}$ beam of $\mathrm{Bi}^{3+}$ ions was employed to analyse the composition distribution by acquiring the mass-to-charge ratios $(\mathrm{m} / \mathrm{q})$ of the negatively charged secondary ions, for example, $\mathrm{C}^{-}(m / q=12), \mathrm{S}^{-}(m / q=32),{ }^{16} \mathrm{O}^{-}(m / q=16)$, ${ }^{18} \mathrm{O}^{-}(\mathrm{m} / \mathrm{q}=16)$ from the analysis area $(100 \mu \mathrm{m} \times 100 \mu \mathrm{m})$ centered at the sputtered region. Data acquisition and subsequent data processing and analysis were performed using SurfaceLab 6 software (ION-TOF GmbH).

Data availability. The authors declare that the data supporting the findings of this study are available within the article and its Supplementary Information.

\section{References}

1. Yang, X. N. et al. Nanoscale morphology of high-performance polymer solar cells. Nano Lett. 5, 579-583 (2005).

2. Bredas, J. L., Norton, J. E., Cornil, J. \& Coropceanu, V. Molecular understanding of organic solar cells: the challenges. Acc. Chem. Res. 42, 1691-1699 (2009).

3. Seo, J.-W., Lee, S.-H. \& Lee, J.-Y. Enhancing quantum efficiency of parallel-like bulk heterojunction solar cells. Appl. Phys. Lett. 103, 123301 (2013).

4. Kim, Y. et al. A strong regioregularity effect in self-organizing conjugated polymer films and high-efficiency polythiophene: fullerene solar cells. Nat. Mater. 5, 197-203 (2006).

5. Vithanage, D. A. et al. Visualizing charge separation in bulk heterojunction organic solar cells. Nat. Comm 4, 2334 (2013).

6. Dibb, G. F. A. et al. Influence of doping on charge carrier collection in normal and inverted geometry polymer: fullerene solar cells. Sci. Rep. 3, 3335 (2013).

7. Jeong, S. et al. Nanoimprinting-induced nanomorphological transition in polymer solar cells: enhanced electrical and optical performance. ACS Nano 9, 2773-2782 (2015).

8. Lee, S.-H., Seo, J.-W. \& Lee, J.-Y. Stable inverted small molecular organic solar cells using a p-doped optical spacer. Nanoscale 7, 157-165 (2015).

9. Campoy-Quiles, M. et al. Morphology evolution via self-organization and lateral and vertical diffusion in polymer: fullerene solar cell blends. Nat. Mater. 7, 158-164 (2008)

10. Li, G. et al. 'Solvent annealing' effect in polymer solar cells based on poly (3-hexylthiophene) and methanofullerenes. Adv. Funct. Mater. 17, 1636-1644 (2007).

11. Peet, J. et al. Efficiency enhancement in low-bandgap polymer solar cells by processing with alkane dithiols. Nat. Mater. 6, 497-500 (2007).

12. Hammond, M. R. et al. Molecular order in high-efficiency polymer/fullerene Bulk heterojunction solar cells. Acs Nano 5, 8248-8257 (2011).

13. Norrman, K., Madsen, M. V., Gevorgyan, S. A. \& Krebs, F. C. Degradation patterns in water and oxygen of an inverted polymer solar cell. J. Am. Chem. Soc. 132, 16883-16892 (2010).

14. Alem, S. et al. Degradation mechanism of benzodithiophene-based conjugated polymers when exposed to light in air. ACS Appl. Mater. Interf. 4, 2993-2998 (2012).
15. Lu, L. \& Yu, L. Understanding low bandgap polymer PTB7 and optimizing polymer solar cells based on it. Adv. Mater. 26, 4413-4430 (2014).

16. Li, N. \& Brabec, C. J. Air-processed polymer tandem solar cells with power conversion efficiency exceeding 10\%. Energy Environ. Sci. 8, 2902-2909 (2015).

17. Poulard, C. \& Damman, P. Control of spreading and drying of a polymer solution from Marangoni flows. Europhys. Lett. 80, 64001 (2007).

18. Morita, T. et al. Enhancement of transport characteristics in poly(3hexylthiophene) films deposited with floating film transfer method. Appl. Phys. Express 2, 111502 (2009).

19. Harkins, W. D. The Physical Chemistry of Surface Films (Reinhold, 1952).

20. Dussaud, A. D. \& Troian, S. M. Dynamics of spontaneous spreading with evaporation on a deep fluid layer. Phys. Fluids 10, 23-38 (1998).

21. Demond, A. H. \& Lindner, A. S. Estimatoin of interfacial-tension betwwen organic liquids and water. Environ. Sci. Technol. 27, 2318-2331 (1993).

22. Lyman, W. J., Reehl, W. F. \& Rosenblatt, D. H. Handbook of Chemical Property Estimation Methods: Environmental Behavior of Organic Compounds 2nd edn 960 (American Chemical Society, 1990).

23. Krebs, F. C. Fabrication and processing of polymer solar cells: A review of printing and coating techniques. Sol. Energy Mater. Sol. Cells 93, 394-412 (2009).

24. Moon, J. S., Lee, J. K., Cho, S. N., Byun, J. Y. \& Heeger, A. J. 'Columnlike’ structure of the cross-sectional morphology of bulk heterojunction materials. Nano Lett. 9, 230-234 (2009).

25. Liang, Y. Y. et al. For the bright future-bulk heterojunction polymer solar cells with power conversion efficiency of 7.4\%. Adv. Mater. 22, E135-E138 (2010).

26. Liu, F. et al. Understanding the morphology of PTB7: PCBM blends in organic photovoltaics. Adv. Energy Mater 4, 1301377 (2014).

27. Schmidt, K. et al. A Mechanistic understanding of processing additive-induced efficiency enhancement in bulk heterojunction organic solar cells. Adv. Mater. 26, 300-305 (2014).

28. Abdelsamie, M., Zhao, K., Niazi, M. R., Chou, K. W. \& Amassian, A. In situ UV-visible absorption during spin-coating of organic semiconductors: a new probe for organic electronics and. J. Mater. Chem. C 2, 3373-3381 (2014).

29. Ma, W., Zhang, J. \& Wang, X. Effect of initial polymer concentration on the crystallization of poly (vinylidene fluoride)/poly (methyl methacrylate) blend from solution casting. J. Macromol. Sci. Phys 47, 139-149 (2008).

30. Young, T. H., Huang, J. H. \& Chuang, W. Y. Effect of evaporation temperature on the formation of particulate membranes from crystalline polymers by drycast process. Eur. Polym. J. 38, 63-72 (2002).

31. Deegan, R. D. Pattern formation in drying drops. Phys. Rev. E 61, 475-485 (2000).

32. Peet, J., Cho, N. S., Lee, S. K. \& Bazan, G. C. Transition from solution to the solid state in polymer solar cells cast from mixed solvents. Macromolecules 41, 8655-8659 (2008).

33. de Gans, B. J. \& Schubert, U. S. Inkjet printing of well-defined polymer dots and arrays. Langmuir 20, 7789-7793 (2004).

34. van Franeker, J. J., Turbiez, M., Li, W., Wienk, M. M. \& Janssen, R. A. J. A realtime study of the benefits of co-solvents in polymer solar cell processing. Nat. Commun. 6, 6229 (2015).

35. Heeger, A. J. 25th Anniversary article: bulk heterojunction solar cells: understanding the mechanism of operation. Adv. Mater. 26, 10-28 (2014).

36. Gao, Y. \& Grey, J. K. Resonance chemical imaging of polythiophene/fullerene photovoltaic thin films: mapping morphology-dependent aggregated and unaggregated C = Cspecies. J. Am. Chem. Soc. 131, 9654-9662 (2009).

37. Tsoi, W. C. et al. The Nature of in-plane skeleton raman modes of P3HT and their correlation to the degree of molecular order in P3HT:PCBM blend thin films. J. Am. Chem. Soc. 133, 9834-9843 (2011).

38. Yin, J., Zhou, Y., Lei, T. \& Pei, J. A butterfly-shaped amphiphilic molecule: solution-transferable and free-standing bilayer films for organic transistors. Angew. Chem. Int. Ed. 50, 6320-6323 (2011).

39. Park, J. Y. \& Advincula, R. C. Nanostructuring polymers, colloids, and nanomaterials at the air-water interface through Langmuir and LangmuirBlodgett techniques. Soft Matter 7, 9829-9843 (2011).

40. Osaka, I., Saito, M., Koganezawa, T. \& Takimiya, K. Thiophene-thiazolothiazole copolymers: significant impact of side chain composition on backbone orientation and solar cell performances. Adv. Mater. 26, 331-338 (2014).

41. Ramamurthy, V. \& Venkatesan, K. Photochemical reactions of organic crystals. Chem. Rev. 87, 433-481 (1987).

42. Brown, W. H. \& Poon, T. Introduction to Organic Chemistry 5th edn 1-792 (John Wiley \& Sons, 2012).

43. Mateker, W. R. et al. Molecular packing and arrangement govern the photooxidative stability of organic photovoltaic materials. Chem. Mat 27, 6345-6453 (2015).

44. Zhou, H. et al. High-efficiency polymer solar cells enhanced by solvent treatment. Adv. Mater. 25, 1646-1652 (2013). 
45. Kim, J. et al. Efficient charge extraction in thick bulk heterojunction solar cells through infiltrated diffusion doping. Adv. Energy Mater. 4, 1301502 (2014).

46. Eckenfelder, W. W., Bowers, A. R. \& Roth, J. A. Chemical Oxidation: Technologies for the Nineties. 1-334 (Technomic, 1997).

47. Shoaee, S. \& Durrant, J. R. Oxygen diffusion dynamics in organic semiconductor films. J. Mater. Chem. C 3, 10079-10084 (2015).

48. Jorgensen, M., Norrman, K. \& Krebs, F. C. Stability/degradation of polymer solar cells. Sol. Energy Mater. Sol. Cells 92, 686-714 (2008).

49. Seemann, A., Egelhaaf, H.-J., Brabec, C. J. \& Hauch, J. A. Influence of oxygen on semitransparent organic solar cells with gas permeable electrodes. Org. Electron. 10, 1424-1428 (2009).

50. Choi, H., Kim, H.-B., Ko, S.-J., Kim, J. Y. \& Heeger, A. J. An organic surface modifier to produce a high work function transparent electrode for high performance polymer solar cells. Adv. Mater. 27, 892-896 (2015).

51. Chu, T.-Y. et al. Morphology control in polycarbazole based bulk heterojunction solar cells and its impact on device performance. Appl. Phys. Lett. 98, 253301 (2011).

52. Chang, Y.-M. \& Leu, C.-Y. Conjugated polyelectrolyte and zinc oxide stacked structure as an interlayer in highly efficient and stable organic photovoltaic cells. J. Mater. Chem. A 1, 6446-6451 (2013).

\section{Acknowledgements}

This work was supported by a National Research Foundation of Korea (NRF) grant funded by the Korean Government (MSIP; no. NRF-2015R1A2A2A01006689 and no. NRF-2015M1A2A2057509). We gratefully acknowledge support from the EEWS Research Project of the office of the KAIST EEWS Initiative (EEWS-2016-N11160015). We thank Changyeon Lee for GIWAXS measurement.

\section{Author contributions}

J.N, S.J. and J.L. conceived and designed the experiments and prepared the manuscript. J.N. and S.J. fabricated the spontaneous spreading films and solar cells, and performed PCE and QE measurements. S.J. analysed organic films using SEM, TEM, ToF-SIMS and XPS. J.N. designed the R2R machine and performed the large-scale transfers of the organic thin films. All authors discussed the results and commented on the manuscript.

\section{Additional Information}

Supplementary Information accompanies this paper at http://www.nature.com/ naturecommunications

Competing financial interests: The authors declare no competing financial interests.

Reprints and permission information is available online at http://npg.nature.com/ reprintsandpermissions/

How to cite this article: Noh, J. et al. Ultrafast formation of air-processable and high-quality polymer films on an aqueous substrate. Nat. Commun. 7:12374 doi: $10.1038 /$ ncomms12374 (2016).

(c) (i) This work is licensed under a Creative Commons Attribution 4.0 International License. The images or other third party material in this article are included in the article's Creative Commons license, unless indicated otherwise in the credit line; if the material is not included under the Creative Commons license, users will need to obtain permission from the license holder to reproduce the material. To view a copy of this license, visit http://creativecommons.org/licenses/by/4.0/

C The Author(s) 2016 